\title{
BEAM DECELERATION PERFORMANCE OF THE FERMILAB MAIN INJECTOR
}

\author{
Gerald P. Jackson \\ Pbar Technologies LLC, 396 West Fenton Lane, Suite 601, West Chicago IL 60185 USA \\ Bruce Brown, David Capista, Brian Chase, Joseph Dey, Robert Flora, Consulato Gattuso \\ Fermi National Accelerator Laboratory, P.O. Box 500, Batavia IL 60510 USA
}

\begin{abstract}
The Fermilab Main Injector was designed to accelerate protons and antiprotons from a momentum of $8.889 \mathrm{GeV} / \mathrm{c}$ to $150 \mathrm{GeV} / \mathrm{c}$. In order to study antiproton deceleration, for the ultimate purpose of storing them in a Penning trap, the Main Injector was used to decelerate protons from the injection momentum down to the goal of $2.0 \mathrm{GeV} / \mathrm{c}$. In this paper the operational experience of working with the Main Injector as a decelerator is presented.
\end{abstract}

\section{OBSERVED BEAM LOSS}

On October 12, 2000 the first systematic attempt to decelerate protons in the Main Injector down to a momentum of $2.0 \mathrm{GeV} / \mathrm{c}$ occurred. Beam was observed down to a momentum of $3.0 \mathrm{GeV} / \mathrm{c}$, with some peculiar structure in the beam loss pattern. The RF system used for deceleration was the $2.5 \mathrm{MHz}$ coalescing system [1]. In this paper much of the beam loss is explained in terms of loss of longitudinal bucket area.

At the very end of the study period the beam survival during the ramp was observed with four different $2.5 \mathrm{MHz}$ RF system voltage amplitude curves. The purpose of this effort was to ascertain if the observed rate of beam loss was due to loss of bucket area during deceleration or due to transverse problems such as incorrect closed orbits, tunes, and chromaticity. The beam survival observation are shown in figure 1.

The green curves (the curves which fall to zero) are of the control system parameter I:IBEAMS, which is the beam intensity in units of $10^{10}$ protons. The plot ranges from 0 to 16 . The fact that one trace, which happened to correspond to the requested $2.5 \mathrm{MHz} \mathrm{RF}$ system voltage amplitude of $25 \mathrm{kV}$, has a lower initial intensity has nothing to do with the measurement conditions. It just happened to be a low pulse out of the Booster accelerator.

The red curves (the curves which start flat and gently rise before quickly dropping) are the $2.5 \mathrm{MHz} \mathrm{RF}$ system voltage amplitude in units of kilovolts. The name of the parameter is I:H28SUM, and it ranges from 0 to 40 . Note that on two traces one of the cavities in the tunnel lost its voltage for approximately one second before resetting.

The blue curve (the curve which begins to smoothly rise at the very end of the plot) is the measured momentum in the Main Injector deceleration ramp in units of $\mathrm{GeV} / \mathrm{c}$. The parameter is called I:MMPRQ, and represents the central momentum of the Main Injector as calculated by the MECAR power supply feedback system. The momentum range on the plot is 1 to $9 \mathrm{GeV} / \mathrm{c}$.

Finally, the black curve (the curve that is the highest value of all curves after eight seconds) is I:VDSPFO, which is the RF frequency of the $53 \mathrm{MHz}$ RF system minus $50 \mathrm{MHz}$. The injection frequency is $52,811,400$ $\mathrm{Hz}$, and the expected frequency at $2 \mathrm{GeV} / \mathrm{c}$ is approximately $48 \mathrm{MHz}$. Therefore, the range of this parameter on the plot is $-4,000,000$ to $+4,000,000 \mathrm{~Hz}$. Note that the frequency was limited to values above that corresponding to $2.8 \mathrm{GeV} / \mathrm{c}$. This temporary limitation was introduced due to the fact that the beam synchronization system was not tracking the RF frequency below $50 \mathrm{MHz}$, causing errant pulses to be emitted into various subsystems such as the Tevatron injection kickers.

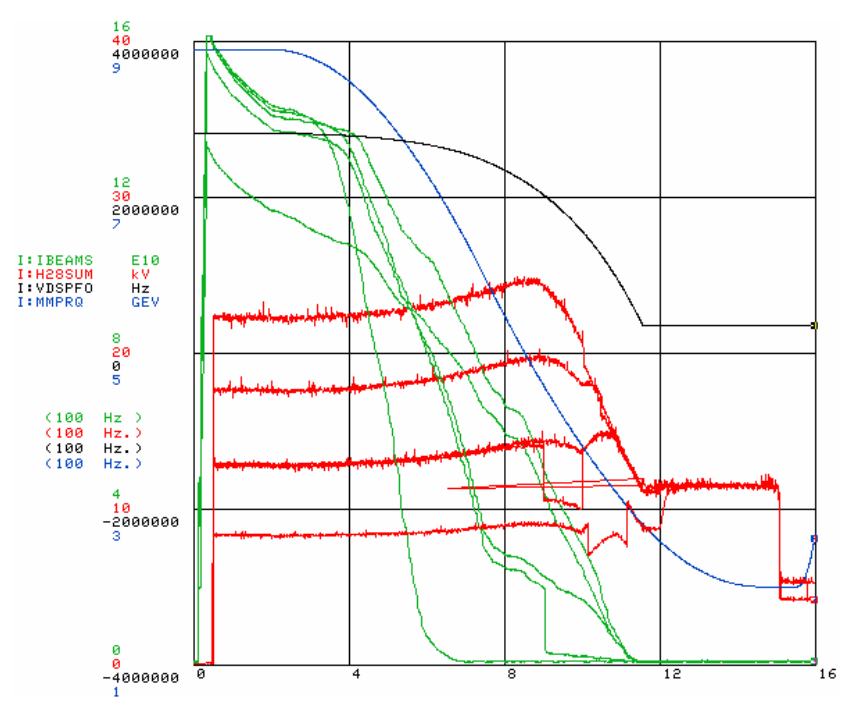

Figure 1: Proton intensity (green) as a function of time for four different RF amplitude curves (red). The RF voltage settings used were $25,20,15$, and $10 \mathrm{kV}$. Note that the beam falls out completely by 7 seconds with an $\mathrm{RF}$ voltage request of $10 \mathrm{kV}$. There are two sets of traces for the $15 \mathrm{kV} \mathrm{RF}$ amplitude request, in which one is normal and the other had an RF station drop out during the ramp. The fact that most of the remaining beam fell out of the Main Injector after the voltage drop is a clear indication that loss of bucket area is dominating the beam loss during deceleration.. 


\section{SIMULATIONS}

Using the program Microsoft Excel, a simple simulation was used to reproduce the data in figure 1 . The results are summarized in the figures in this section. Each figure contains curves which correspond directly to individual curves found in figure 1 .

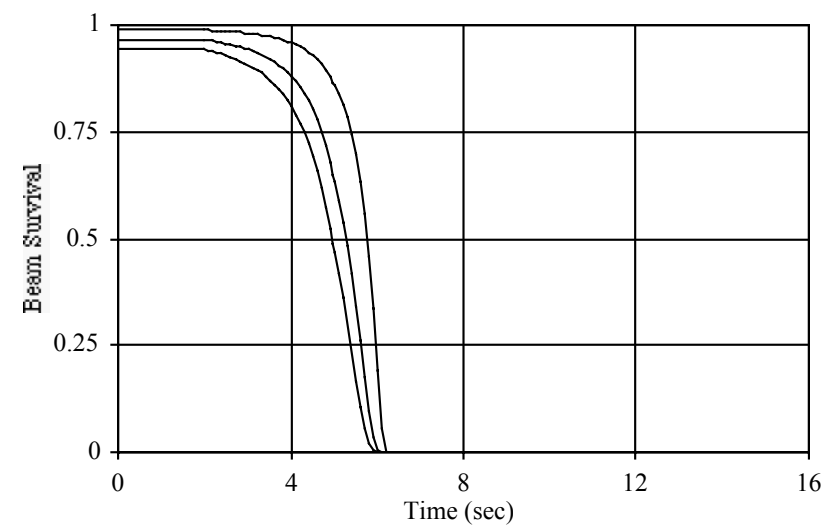

Figure 2: Calculated beam survival during deceleration when the $2.5 \mathrm{MHz}$ RF system is set to a peak voltage of 9 $\mathrm{kV}$. This corresponds to the measured trace in which an amplitude of $10 \mathrm{kV}$ was requested. In this simulation the longitudinal emittance of the coalesced beam was set to $3.0 \mathrm{eV}$-sec for the middle curve, and the lower and upper curves are for 5 and $1 \mathrm{eV}$-sec respectively. Note the quantitative agreement between this simulation result and the observed beam loss in figure 1 .

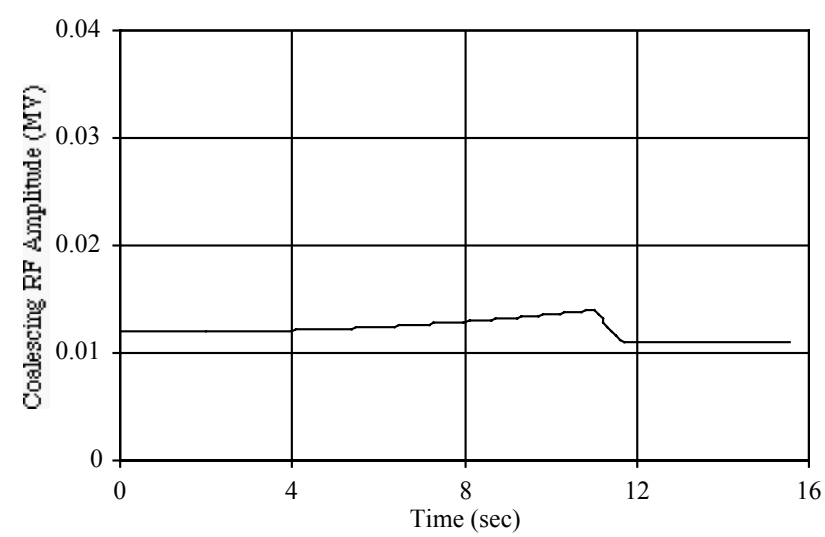

Figure 3: RF voltage curve used in the simulation for the condition in which $15 \mathrm{kV}$ was requested in figure 1 . This has been tuned so that it approximates the observed cavity voltage.

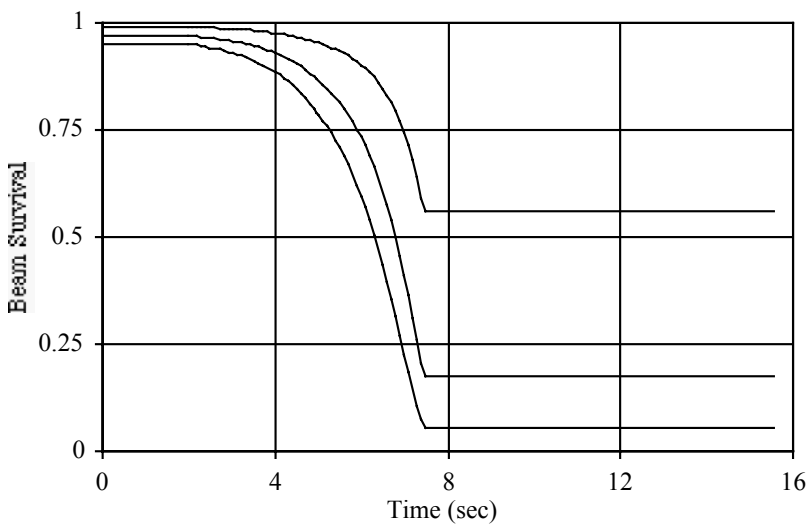

Figure 4: Beam survival during deceleration when the $2.5 \mathrm{MHz} \mathrm{RF}$ system is matched to the observed RF voltage amplitude in the Main Injector when $15 \mathrm{kV}$ is requested. In this simulation the longitudinal emittance of the coalesced beam was set to $3.0 \mathrm{eV}$-sec for the middle curve, and the lower and upper curves are for 5 and $1 \mathrm{eV}$-sec respectively.

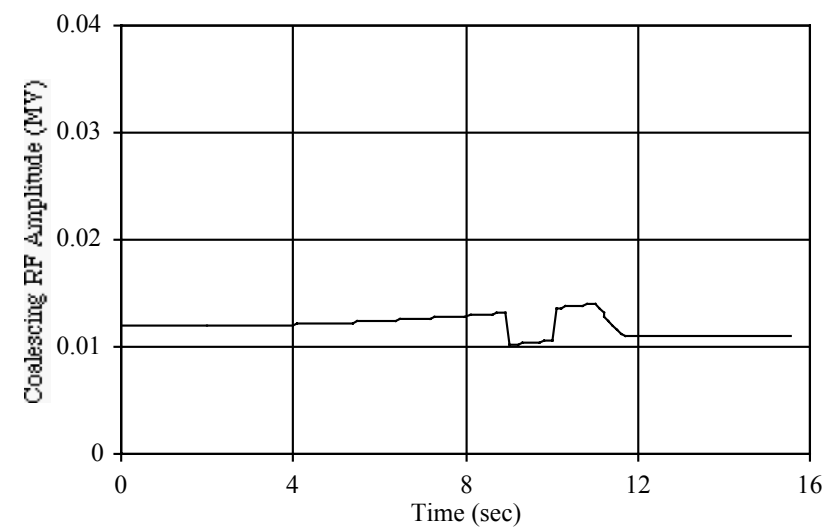

Figure 5: RF voltage amplitude curve used in the simulation for the condition in which $15 \mathrm{kV}$ was requested. This has been tuned so that it approximates the observed cavity voltage, including the cavity trip event that is evident in figure 1 .



Figure 6: Beam survival during deceleration when $15 \mathrm{kV}$ is requested, but when a cavity trip also occurs during deceleration. Note that the immediate drop in beam survival precisely mimics the observation in figure 1 . 


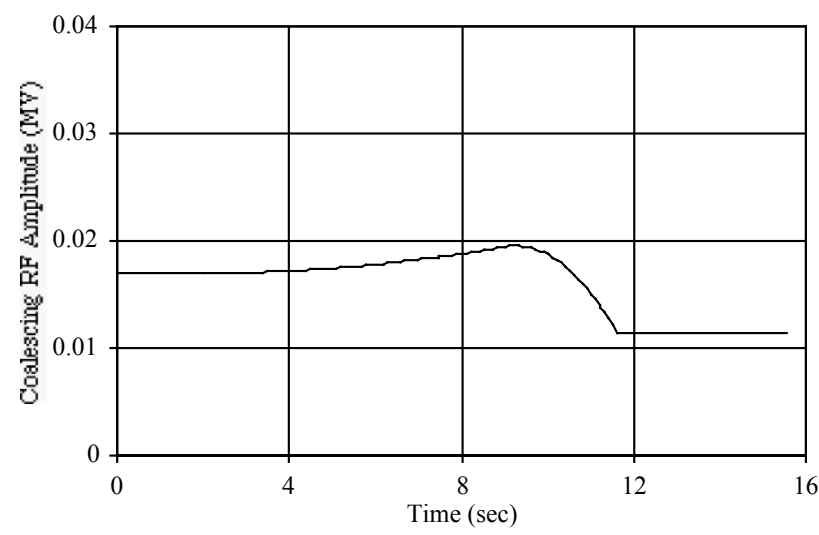

Figure 7: RF voltage amplitude curve used in the simulation for the condition in which $20 \mathrm{kV}$ was requested. This has been tuned so that it approximates the observed cavity voltage in figure 1 .

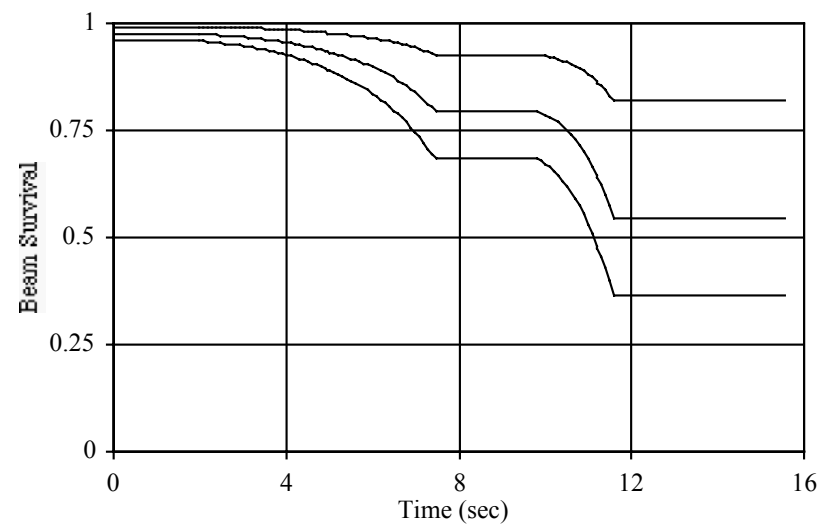

Figure 8: Beam survival during deceleration when $20 \mathrm{kV}$ is requested, but when a cavity trip also occurs during deceleration. This is the first time that comparisons with measured figure 1 data in the Main Injector are not so precise. At this point the background beam lifetime is clearly the dominant source of beam loss.

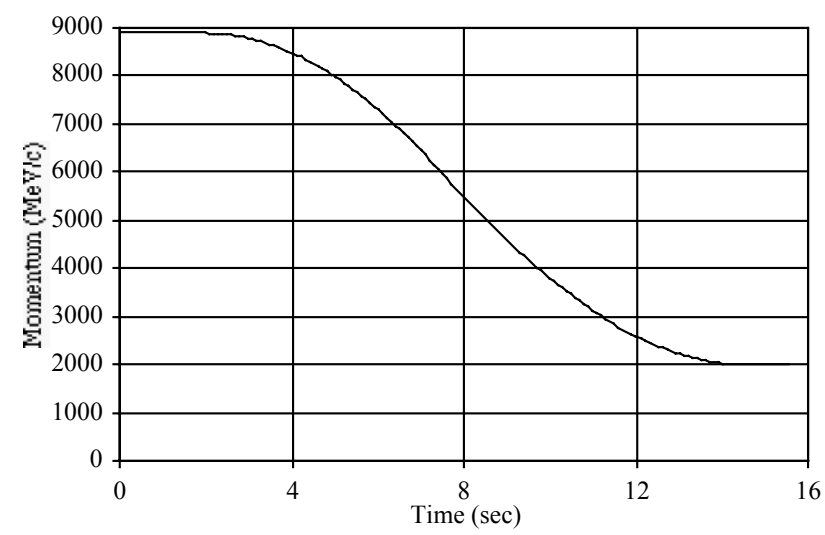

Figure 9: Momentum ramp of the Main Injector during the deceleration cycles.

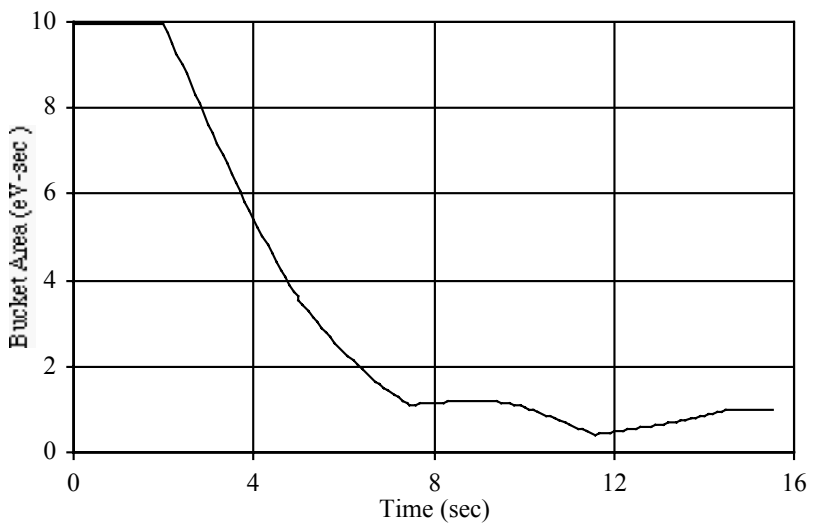

Figure 10: Bucket area vs. time for the RF amplitude curve corresponding to the $20 \mathrm{kV}$ request that was used for most of the study period displayed in figure 1 .

\section{FUTURE DIRECTIONS}

In order to speed up the ramp rate and decelerate with a better bucket area to bunch longitudinal emittance ratio, experiments are underway to use the Main Injector 53 $\mathrm{MHz}$ RF system. Because that system is intrinsically very narrow band, schemes such as transient filling the cavities turn-by-turn and harmonic jumping are being investigated.

\section{REFERENCES}

[1] I. Kourbanis and D. Wildman, Proc. 1999 Part. Acc. Conf., pg. 2840. 